\title{
ON AN INTEGRAL TRANSFORM
}

\section{NAYLOR}

University of Western Untario

London, Ontario, Canada

(Received November 26, 1985)

ABSTRACT. This paper establishes properties of a convolution type integral transform whose kernel is a Macdonald type Bessel function of zero order. An inversion formula is developed and the transform is applied to obtain the solution of some related integral equations.

KEY WORDS AND PHRASES. Integral Transforms, Integral Equations, Bessel Functions. 1980 AMS MATHEMATICS SUBJECT CLASSIFICATION CODES. 44A15, 45H05, 33A40.

1. THE TRANSFORM AND THE FORMULA OF INVERSION.

This paper investigates properties of the integral transform defined by the equation

$$
F(x)=\int_{-\infty}^{\infty} f(t) k_{0}(|x-t|) d t
$$

whers $-\infty-x=\infty$ and $K_{0}$ denotes the Macdonald type Bessel function of zero order. A number of results concerning the above transform are obtained, including a formula of inversion and a Parseval type equation, and the transform is applied to obtain the solution of some associated integral equations one of which is of the Wiener-Hopf type.

A formula of inversion for the transform (1.1) may be derived with the aid of the equation

$$
\int_{-\infty}^{\infty} K_{0}(|x-t|) K_{0}(|x-y|) d x=\frac{\pi^{2}}{2} e^{-|t-v|}
$$

This result may be established by applying a Parseval type theorem in the theory of Fourier integrals. This is carried out in the final section of this paper which contains a short list of transforms together with an outline of some methods that can be adopted for deriving them

The following theorem will be proved:

THEOREM. Let $f(x)$ be continuous and suppose that $f(x) e^{-|x|} \varepsilon L(-\infty, \infty)$ then the integral transform defined by equation (1.1) possesses the inverse

$$
f(x)=-\frac{1}{\pi^{2}}\left(D^{2}-1\right) \int_{-\infty}^{\infty} F(t) K_{0}(|x-t|) d t
$$

where $D=d / d x$. 
To establish this result we consider the expression

$$
\int_{-\infty}^{\infty} f(t) d t \int_{-\infty}^{\infty} K_{0}(|x-t|) K_{0}(|x-y|) d x
$$

We first verify that this repeated integral is absolutely convergent. Since the Bessel functions appearing in (1.4) are positive the modulus of the expression (1.4) cannot exceed that of the integral

$$
\int_{-\infty}^{\infty}|f(t)| d t \int_{-\infty}^{\infty} K_{0}(|x-t|) K_{0}(|x-y|) d x=\frac{\pi^{2}}{2} \int_{-\infty}^{\infty}|f(t)| e^{-|y-t|} d t
$$

after substituting the formula (1.2). The integral on the right hand side of (1.5) is equal to the expression

$$
\pi^{2}\left[e^{-y} \int_{-\infty}^{y}|f(t)| e^{t} d t+e^{y} \int_{y}^{\infty}|f(t)| e^{-t} d t\right]
$$

This expression is finite since $f(x) e^{-|x|} \varepsilon L(-\infty, \infty)$. The order of integration appearing in (1.4) may now be reversed. This yields the equation

$$
\int_{-\infty}^{\infty} f(t) d t \int_{-\infty}^{\infty} K_{0}(|x-t|) K_{0}(|x-y|) d x=\int_{-\infty}^{\infty} K_{0}(|x-y|) d x \int_{-\infty}^{\infty} f(t) K_{0}(|x-t|) d t
$$

so that, by $(1.1)$ and $(1.2)$,

$$
\pi^{2} \int_{-\infty}^{\infty} f(t) e^{-|y-t|} d t=\int_{-\infty}^{\infty} F(x) K_{o}(|x-y|) d x
$$

Now if $\lambda$ is a positive constant and since $f(x)$ is continuous it can be shown that

$$
-2 \lambda f(y)=\left(\frac{d^{2}}{d y^{2}}-\lambda^{2}\right) \int_{-\infty}^{\infty} f(t) e^{-\lambda|y-t|} d t
$$

To obtain this result the domain of integration is decomposed into the parts $(-\infty, y)$ and $(y, \alpha)$ and the differentiation carried out. On setting $\lambda=1$ in (1.7) and applying this result to equation (1.6) we obtain the desired formula (1.3), after redefining the variables.

For the remainder of this section it will be supposed that the function $f(x)$ together with its derivatives of the first and second order are continuous and of modulus less than $C e^{c|x|}$ where $C$, $c$ are constants and $-1<c<1$, $C>0$. It will then follow that the transform $F(x)$ is $0\left(e^{c|x|}\right)$ as $|x| \rightarrow \infty$. This result may be deduced from (1.1) by inserting the stated bound on $f(x)$ together with the bound, obtained in section 5 of the paper, that

$$
K_{0}(x) \leqq(\pi / 2 x)^{\frac{1}{2}} e^{-x}
$$

whenever $x>0$. The substitution of these bounds into (1.1) yields the inequality

$$
|F(x)| \leqq C^{\prime} \int_{-\infty}^{\infty} e^{-|t|+c|x-t|} \frac{d t}{|t|^{\frac{1}{2}}}
$$

where $C^{\prime}$ is a constant. On decomposing the domain of integration into the segments $(-\infty, x)$ and $(x, \infty)$ we find that 


$$
|F(x)| \leqq C^{\prime} e^{c x} \int_{-\infty}^{x} e^{-c t-|t|} \frac{d t}{|t|^{\frac{1}{2}}}+C^{\prime} e^{-c x} \int_{x}^{\infty} e^{c t-|t|} \cdot d t
$$

Since $-1<\mathrm{c}<1$ the integrals appearing in the preceding inequality are convergent and the first also exists as $|x| \rightarrow \infty$, so that $F(x)=0\left(e^{c|x|}\right)$ as stated. It is clear

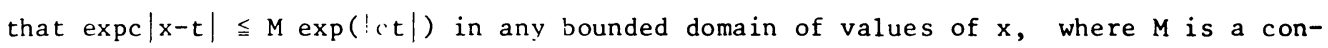
stant, so that, by (1.3), the integral defining the transform $F(x)$ is uniformly convergent in any such domain.

Since it is assumed that the derivatives $f^{\prime}(x)$ and $f^{\prime \prime}(x)$ satisfy the same types of bounds as $f(x)$ itself, then the integrals defining their transforms will also converge uniformly so that differentiation through the integral sign is permissable. Therefore we obtain the equations

$$
\begin{aligned}
& F^{\prime}(x)=\int_{-\infty}^{\infty} f^{\prime}(t) K_{0}(|x-t|) d t \\
& F^{\prime \prime}(x)=\int_{-\infty}^{\infty} f^{\prime \prime}(t) K_{0}(|x-t|) d t
\end{aligned}
$$

The transforms $F(x), F^{\prime}(x), F^{\prime \prime}(x)$ are each $O\left(e^{c|x|}\right)$ as $|x| \rightarrow \infty$ so that the integrals

$$
\int_{-\infty}^{\infty} F(t) K_{0}(|x-t|) d t, \quad \int_{-\infty}^{\infty} F^{\prime \prime}(t) K_{0}(|x-t|) d t
$$

converge uniformly in any bounded domain of values of $x$. Therefore

$$
\frac{d^{2}}{d x^{2}} \int_{-\infty}^{\infty} F(t) K_{0}(|x-t|) d t=\int_{-\infty}^{\infty} F^{\prime \prime}(t) K_{0}(|x-t|) d t
$$

To verify results like this and like (1.9) and (1.10), the variable of integration is temporarily replaced by $(x-t)$. After the differentiation with respect to $x$ has been carried out the original variable of integration can be restored. Therefore for a function $f(x)$ which is such that $f, f^{\prime}, f^{\prime \prime}$ are continuous and are $0\left(e^{c|x|}\right)$ as $|x| \rightarrow \infty$, where $-1<c<1$, the inversion formula (1.3) can be stated as the equation

$$
f(x)=-\frac{1}{\pi} \int_{-\infty}^{\infty}\left[F^{\prime \prime}(t)-F(t)\right] K_{0}(|x-t|) d t
$$

\section{A RELATED INTEGRAL TRANSFOPM.}

In this section the inversion formula already derived for the transform (1.1) will be applied to obtain a formula of inversion for the related transform defined by the equation

$$
F_{1}(x)=\int_{0}^{\infty} f(t) K_{0}(|x-t|) d t
$$

in which $\mathrm{F}_{1}(\mathrm{x})$ is supposed defined for $\mathrm{x}>0$ only. If $(2.1)$ is regarded as an integral equation, it is of the kind to which the Wiener-Hopf procedure, Noble [1], could be applied but the method proposed here is more direct.

In order to apply the formula (1.3) it is necessary to obtain the values of the integral occurring on the right hand side of equation (2.1) for negative values of $x$. 
These values can be expressed in terms of the values of the same integral for positive values of $x$, that is, in terms of the given function $F_{1}(x)$. This may be carried out with the aid of the transform of the function $t^{-\frac{1}{2}} e^{-t}(t+x)^{-1} H(t)$, where $H(t) d e-$ notes the Heaviside unit function, which is calculated in section 5 of the paper, where it appears as equation (5.7). On multiplying equation (5.7) by $f(t)$ and integrating it follows that

$$
\begin{aligned}
\int_{0}^{\infty} f(t) K_{0}(x+t) d t & =\frac{1}{\pi} x^{\frac{1}{2}} e^{-x} \int_{0}^{\infty} f(t) d t \int_{0}^{\infty} \frac{e^{-y_{K}}(|t-y|) d y}{y^{\frac{1}{2}}(x+y)} \\
& =\frac{1}{\pi} x^{\frac{1}{2}} e^{-x} \int_{0}^{\infty} \frac{e^{-y_{F}}(y) d y}{y^{\frac{1}{2}}(x+y)}
\end{aligned}
$$

after changing the order of integration and substituting (2.1). This result applies for $x, 0$. It follows, if $x$ is negative, that

$$
\int_{0}^{\infty} f(t) K_{0}(|x-t|) d t=\frac{1}{\pi}|x|^{\frac{1}{2}} e^{+x} \int_{0}^{\infty} \frac{e^{-y} F_{1}(y) d y}{y^{\frac{1}{2}}(y-x)}
$$

The equations (2.1) and (2.3), when taken together, form an equation of the same type as (1.1), provided we define $f(t)=0$ therein whenever $t<0$. On applying the inversion formula (1.3) to (2.1) and (2.3) we find the solution of equation (2.1). This procedure yields the formula

$$
\begin{aligned}
f(x)=-\frac{1}{\pi}\left(D^{2}-1\right) & \int_{0}^{\infty} F_{1}(t) K_{0}(|x-t|) d t \\
& -\frac{1}{\pi^{2}}\left(D^{2}-1\right) \int_{-\infty}^{0}|t|^{\frac{1}{2}} e^{t} K_{0}(|x-t|) d t \int_{0}^{\infty} \frac{e^{-y_{F}} F_{1}(y) d y}{y^{\frac{1}{2}}(y-t)}
\end{aligned}
$$

It can be verified, after performing some calculation, that the above solution agrees with that obtained by Noble [2] using the Wiener-Hopf technique.

3. SOLUTION OF AN INTEGRAL EQUATION.

The transform defined by equation (1.1) was introduced to study certain integral equations whose kernels involved the Bessel function $k_{0}(|x-t|)$. An equation of this type is that given by the equation

$$
g_{1}(x)=\int_{-\infty}^{\infty} f(t) \cosh \lambda(x-t) K_{0}(|x-t|) d t
$$

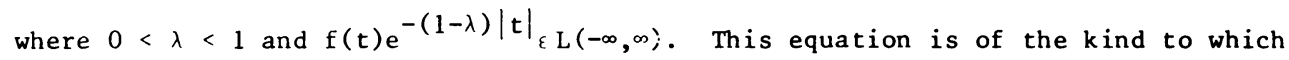
the method of Fourier integrals can be applied, however since the Fourier transform of the kernel is not of a simple form the following alternative method, which is based on the identity (1.2) is suggested. We consider first the integral

$$
I=\int_{-\infty}^{\infty} \cosh \lambda t \sinh \lambda(x-t) K_{0}(j t \mid) K_{0}(|x-t|) d t
$$

On replacing the variable of integration by $(x-t)$ we find the equation

$$
I=\int_{-\infty}^{\infty} \cosh \lambda(x-t) \sinh \lambda t K_{0}(|t|) K_{0}(|x-t|) d t
$$


Upon taking the sum of (3.2) and (3.3) and simplifying the integrand we find that

$$
2 I=\sinh \lambda x \int_{-\infty}^{\infty} K_{0}(|t|) K_{0}(|x-t|) d t=\frac{\pi^{2}}{2} e^{-|x|} \sinh \lambda x
$$

by virtue of $(1.2)$. Therefore

$$
\int_{-\infty}^{\infty} \cosh \lambda(x-t) \sinh \lambda t K_{0}(|t|) k_{0}(|x-t|) d t=\frac{\pi^{2}}{4} e^{-|x|} \sinh \lambda x
$$

After replacing $t$ by $(t-a)$ and setting $b=x+a$ we find the equation

$$
\int_{-\infty}^{\infty} \cosh \lambda(b-t) \sinh \lambda(t-a) k_{0}(|t-a|) K_{0}(|t-b|) d t=\frac{\pi^{2}}{4} e^{-|a-b|} \sinh \lambda(b-a)
$$

The equation (3.1) is now multiplied by $\sinh \lambda(x-a) K_{0}(|x-a|)$ and integrated with respect to $x$. On changing the order of integration and using (3.6) we $f$ ind that

$$
\begin{aligned}
\int_{-\infty}^{(x)} g_{1}(x) \sinh \lambda & (x-a) K_{0}(|x-a|) d x=\frac{\pi^{2}}{4} \int_{-\infty}^{\infty} f(t) e^{-|t-a|} \sinh \lambda(t-a) d t \\
& =\frac{\pi^{2}}{8} \int_{-\infty}^{\infty} f(t)\left[e^{-(1-\lambda)|t-a|}-e^{-(1+\lambda)|t-a|}\right] \operatorname{sgn}(t-a) d t
\end{aligned}
$$

Now it can be verified if $\mathrm{f}(\mathrm{t})$ is continuous that

$$
D \int_{-\infty}^{\infty} f(t) e^{-\lambda|x-t|} d t=\lambda \int_{-\infty}^{\infty} f(t) e^{-\lambda|x-t|} \operatorname{sgn}(t-x) d t
$$

where $D=d / d x$. If this result be combined with that of equation (1.7) we obtain the formula

$$
f^{\prime}(x)=-\frac{1}{2}\left(D^{2}-\lambda^{2}\right) \int_{-\infty}^{\infty} f(t) e^{-\lambda|x-t|} \operatorname{sgn}(t-x) d t
$$

When this result is applied in succession to equation (3.7) with $\lambda$ replaced in turn by $(1-\lambda)$ and $(1+\lambda)$ we find, after redefining the variables, that

$$
f^{\prime}(x)=\frac{1}{\lambda \pi^{2}}\left[D^{2}-(1-\lambda)^{2}\right]\left[D^{2}-(1+\lambda)^{2}\right] \int_{-\infty}^{\infty} g_{1}(t) \sinh \lambda(t-x) K_{0}(|t-x|) d t
$$

This equation determines the function $f(x)$ up to an additive constant which could be fixed by imposing the condition, obtained from (3.1) with $x$ set equal to zero, that

$$
g_{1}(0)=\int_{-\infty}^{\infty} f(t) \cosh \lambda t K_{0}(|t|) d t
$$

The formula (3.6) can also be used to obtain the inversion formula for the equation

$$
g_{2}(x)=\int_{-\infty}^{\infty} f(t) \sinh \lambda(x-t) K_{0}(|x-t|) d t
$$

The solution of this equation is given by the formula

$$
f^{\prime}(x)=-\frac{1}{\lambda \pi^{2}}\left[D^{2}-(1-\lambda)^{2}\right]\left[D^{2}-(1+\lambda)^{2}\right] \int_{-\infty}^{\infty} g_{2}(t) \cosh \lambda(t-x) K_{0}(|t-x|) d t
$$

If we replace $\lambda$ by $i \lambda$ in the above formulas we obtain the solutions of the equations (3.1), (3.11) in which the hyperbolic functions are replaced by trigonometric functions. Thus if 


$$
g_{3}(x)=\int_{-\infty}^{\infty} f(t) \cos \lambda(x-t) K_{0}(|x-t|) d t
$$

then it is found from (3.10) after replacing $\lambda$ by $i \lambda$ and regrouping the factors in the differential operator that

$$
f^{\prime}(x)=\frac{1}{\lambda \pi^{2}}\left[\lambda^{2}+(D+1)^{2}\right]\left[\lambda^{2}+(D-1)^{2}\right] \int_{-\infty}^{\infty} g_{3}(t) \sin \lambda(t-x) K_{0}(|t-x|) d t
$$

4. CONVOLUTION INTEGRALS.

Since the integral (1.1) is itself of the convolution type it is possible to formulate convolution type theorems connecting the transforms of pairs of functions. Let $g(x)$ be continuous and $0\left(e^{\mu|x|}\right)$ as $|x| \rightarrow \infty$ where $-1<\mu<1$ then its transform $G(x)$ exists and is $0\left(e^{\mu|x|}\right)$ as $|x| \rightarrow \infty$. Therefore, if $c+\mu<0$, we may form the integral

$$
\begin{aligned}
\int_{-\infty}^{\infty}\left[F^{\prime \prime}(x)-F(x)\right] G(x-y) d x & =\int_{-\infty}^{\infty}\left[F^{\prime \prime}(x)-F(x)\right] d x \int_{-\infty}^{\infty} g(t) K_{0}(|x-y-t|) d t \\
& =\int_{-\infty}^{\infty} g(t) f(y+t) d t
\end{aligned}
$$

after reversing the order of integration and appealing to the formula (1.11). Similarly we find the equation

$$
\int_{-\infty}^{\infty}\left[F^{\prime \prime}(x)-F(x)\right] G(y-x) d x=\int_{-\infty}^{\infty} g(t) f(y-t) d t
$$

Alternatively we may consider the convolution integral

$$
\begin{aligned}
\int_{-\infty}^{\infty} F(x) g(x-y) d x & =\int_{-\infty}^{\infty} g(x-y) d x \int_{-\infty}^{\infty} f(t) K_{0}(|x-t|) d t \\
& =\int_{-\infty}^{\infty} f(t) G(t-y) d t
\end{aligned}
$$

The above formulas may be used to express the transforms of products like $t f(t)$, $t f^{\prime}(t), t f^{\prime \prime}(t)$ in terms of the transform $F(x)$. Thus if we apply formula (4.3) to the function $g(x)=e^{-|x|} \operatorname{sgnx}$, which by the formula (5.5), possesses the transform given by $G(x)=2 x_{0}(|x|)$, we obtain the equation

$$
\begin{aligned}
\int_{-\infty}^{\infty} F(x) e^{-|x-y|} \operatorname{sgn}(x-y) d x & =2 \int_{-\infty}^{\infty} f(t)(t-y) K_{0}(|t-y|) d t \\
& =-2 y F(y)+2 \int_{-\infty}^{\infty} t f(t) K_{0}(|t-y|) d t
\end{aligned}
$$

after recalling the definition (1.1). It follows that the transform of the product $t f(t)$ is given by the equation

$$
\int_{-\infty}^{\infty} t f(t) K_{0}(|y-t|) d t=y F(y)+\frac{1}{2} \int_{-\infty}^{\infty} F(x) e^{-|x-y|} \operatorname{sgn}(x-y) d x
$$

If this relation be applied to the function $f^{\prime}(t)$ we $f$ ind, since $F^{\prime}(y)$ is the transform of $f^{\prime}(t)$, that 


$$
\begin{aligned}
\int_{-\infty}^{\infty} t f^{\prime}(t) K_{0}(|y-t|) d t & =y F^{\prime}(y)+\frac{1}{2} \int_{-\infty}^{\infty} F^{\prime}(x) e^{-|x-y|} \operatorname{sgn}(x-y) d x \\
& =y F^{\prime}(y)-F(y)+\frac{1}{2} \int_{-\infty}^{\infty} F(y-x) e^{-|x|} d x
\end{aligned}
$$

after an integration by parts.

Similarly we find on replacing $f(t)$ by $f^{\prime}(t)$ in (4.5) that

$$
\int_{-\infty}^{\infty} t f^{\prime \prime}(t) K_{0}(|y-t|) d t=y F^{\prime \prime}(y)-F^{\prime}(y)-\frac{1}{2} \int_{-\infty}^{\infty} F(y-x) e^{-|x|} \operatorname{sgn} x d x
$$

after an integration by parts.

On subtracting $(4.6),(4.4)$ it follows that

$$
\int_{-\infty}^{\infty} t\left[f^{\prime \prime}(t)-f(t)\right] K_{0}(|y-t|) d t=y F^{\prime \prime}(y)-y F(y)-F^{\prime}(y)
$$

Since $F^{\prime}(y)$ is the transform of $f^{\prime}(t)$, the equation (4.7) is equivalent to the equation

$$
\int_{-\infty}^{\infty}\left[t f^{\prime \prime}(t)+f^{\prime}(t)-t f(t)\right] K_{0}(|y-t|) d t=y F^{\prime \prime}(y)-y F(y)
$$

5. DERIVATION OF SOME PARTICULAR TRANSFORMS.

In this section some methods are described by means of which the transforms of some simple functions may be calculated. The first such method is to appeal to the Parseval formula in the theory of Fourier integrals which states, Titchmarsh [3], that

$$
\int_{-\infty}^{\infty} f(t) g(-t) d t=\frac{1}{2 \pi} \int_{-\infty}^{\infty} F_{0}(s) G_{0}(s) d s
$$

where $F_{0}, G_{0}$ denote the Fourier transforms of $f, g$. We first set $g(t)=K_{0}(|x+t|)$ so that

$$
\begin{aligned}
G_{0}(s) & =\int_{-\infty}^{\infty} e^{i s t} K_{0}(|x+t|) d t=e^{-i s x} \int_{-\infty}^{\infty} e^{i s t} K_{0}(|t|) d t \\
& =\frac{\pi e^{-i s x}}{\left(1+s^{2}\right)^{\frac{1}{2}}}
\end{aligned}
$$

by Watson [4], p. 388. On inserting this choice of $g, G_{0}$ in (5.1) we find the equation

$$
\int_{-\infty}^{\infty} f(t) k_{0}(|x-t|) d t=\frac{1}{2} \int_{-\infty}^{\infty} \frac{e^{-i s x_{F}}(s) d s}{\left(1+s^{2}\right)^{\frac{1}{2}}}
$$

where

$$
F_{0}(s)=\int_{-\infty}^{\infty} e^{i s t} f(t) d t
$$

To obtain the basic formula (1.2) we set $f(t)=K_{0}(|t|)$ in (5.2). Since $F_{0}(s)=\pi\left(1+s^{2}\right)^{-\frac{1}{2}}$ we find that

$$
\int_{-\infty}^{\infty} k_{0}(|t|) K_{0}(|x-t|) d t=\frac{\pi}{2} \int_{-\infty}^{\infty} \frac{e^{-i s x} d s}{1+s^{2}}
$$


The integral on the right hand side of this equation is elementary and is equal to $\pi e^{-|x|}$, therefore,

$$
\int_{-\infty}^{\infty} K_{0}(|t|) K_{0}(|x-t|) d t=\pi_{2}^{2} e^{-|x|}
$$

The equation (1.2) follows from the preceding equation after making a simple change of variable and redefining the constants.

Next we consider the function $f(t)=e^{-|t|}$ whose Fourier transform is the function $F_{0}(s)=2\left(1+s^{2}\right)^{-1}$. The substitution of this pair into (5.2) yields the equation

$$
\int_{-\infty}^{\infty} e^{-|t|} K_{0}(|x-t|) d t=\int_{-\infty}^{\infty} \frac{e^{-i s x} d s}{\left(1+s^{2}\right)^{3 / 2}}=2|x| K_{1}(|x|)
$$

where we have used the formula, Watson [4], p. 172,

$$
\mathrm{K}_{\mathrm{u}}(\mathrm{x})=\frac{1}{\sqrt{\pi}}(2 / \mathrm{x})^{\mathrm{u}} \Gamma\left(\mathrm{u}+\frac{1}{2}\right) \int_{0}^{\infty} \frac{\cos (\mathrm{s} x) \mathrm{ds}}{\left(1+\mathrm{s}^{2}\right)^{\mathrm{u}+\frac{1}{2}}}
$$

which applies whenever $\operatorname{Re}(u)>-\frac{1}{2}$.

Further transforms may be constructed by using the fact that $F^{\prime}(x)$ is the transform of $f^{\prime}(t)$. Thus from the pair appearing in (5.4) we obtain by differentiation the transform

$$
\int_{-\infty}^{\infty} e^{-|t|} K_{0}(|x-t|) \operatorname{sgn} t d t=2 x K_{0}(|x|)
$$

after using the formula $(d / d x)\left(x K_{1}(x)\right)=-x K_{0}(x)$.

The transform of the product $t_{0}(|t|)$ is the integral

$$
J=\int_{-\infty}^{\infty} t K_{0}(|t|) K_{0}(|x-t|) d t
$$

On replacing $t$ by $(x-t)$ we find that

$$
J=\int_{-\infty}^{\infty}(x-t) k_{0}(|t|) k_{0}(|x-t|) d t
$$

It follows after adding the two expressions for $J$ that

$$
J=\frac{x}{2} \int_{-\infty}^{\infty} K_{0}(|t|) K_{0}(|x-t|) d t=\frac{\pi^{2} x}{4} e^{-|x|}
$$

by (5.3). Therefore the transform of $t_{0}(|t|)$ is $\frac{1}{4} \pi^{2} x e^{-|x|}$.

Another method of calculating the transforms is to make use of the formula, Lowndes [5],

$$
\mathrm{K}_{\mathrm{o}}(|\mathrm{x}-\mathrm{t}|)=\mathrm{e}^{\mathrm{x}+\mathrm{t}} \int_{\mathrm{u}_{0}}^{\infty} \frac{\mathrm{e}^{-2 \mathrm{u}} \mathrm{du}}{(\mathrm{u}-\mathrm{x})^{\frac{1}{2}}(u-t)^{\frac{1}{2}}}
$$

where $u_{0}$ is the greater of $(x, t)$.

We illustrate the use of the formula (5.6) to calculate the transform of the function $f(t)=t^{-\frac{1}{2}} e^{-t} H(t)$. The resulting integrals are elementary and we obtain the formula

$$
\int_{0}^{\infty} t^{-\frac{1}{2}} e^{-t} K_{0}(|x-t|) d t=\pi(\pi / 2)^{\frac{1}{2}} e^{-x}
$$


valid for $x>0$.

The transform of the function $f(t)=t^{-\frac{1}{2}} e^{-t}(t+z)^{-1}$ required in section 2 can also be calculated from the formula (5.6). On appealing to the result

$$
\int_{0}^{v} \frac{d t}{(t+z) t^{\frac{1}{2}}(v-t)^{\frac{1}{2}}}=\frac{\pi}{z^{\frac{1}{2}}(v+z)^{\frac{1}{2}}}
$$

we $f$ ind that

$$
\begin{aligned}
\int_{0}^{\infty} \frac{e^{-t} K_{0}(|x-t|) d t}{t^{\frac{1}{2}}(t+z)} & =\frac{\pi e^{x}}{\sqrt{z}} \int_{x}^{\infty} \frac{e^{-2 u} d u}{(u-x)^{\frac{1}{2}}(u+z)^{\frac{1}{2}}} \\
& =\frac{\pi e^{z}}{\sqrt{z}} K_{0}(x+z)
\end{aligned}
$$

for $x$ and $z$ positive. If we use $y$ as the variable of integration and redefine the constants we obtain the equation used in section 2 in the form

$$
K_{0}(x+t)=\frac{1}{\pi} x^{\frac{1}{2}} e^{-x} \int_{0}^{\infty} \frac{e^{-y_{K}} K_{o}(|t-y|) d y}{y^{\frac{1}{2}}(x+y)}
$$

The transforms of exponential, hyperbolic and trigonometric functions can be deduced from the Laplace transform result that

$$
\int_{0}^{\infty} \cosh \lambda t K_{0}(t) d t=\frac{\pi}{2}\left(1-\lambda^{2}\right)^{-\frac{1}{2}}
$$

This formula applies for $-1<\lambda<1$ and is given in Oberhettinger [6], p. 151. It follows that

$$
\int_{-\infty}^{\infty} e^{-\lambda t} K_{0}(|t|) d t=\pi\left(1-\lambda^{2}\right)^{-\frac{1}{2}}
$$

so that, after replacing $t$ by $x-t$, we obtain the formula

$$
\int_{-\infty}^{\infty} e^{-\lambda t} K_{0}(|x-t|) d t=\frac{\pi e^{-\lambda x}}{\left(1-\lambda^{2}\right)^{\frac{1}{2}}}
$$

It follows from this result that

$$
\begin{aligned}
& \int_{-\infty}^{\infty} \cosh \lambda t K_{0}(|x-t|) d t=\frac{\pi \cosh \lambda x}{\left(1-\lambda^{2}\right)^{\frac{1}{2}}} \\
& \int_{-\infty}^{\infty} \sinh \lambda t K_{0}(|x-t|) d t=\frac{\pi \sinh \lambda x}{\left(1-\lambda^{2}\right)^{\frac{1}{2}}}
\end{aligned}
$$

Similarly from the Fourier transform formula

$$
\int_{-\infty}^{\infty} e^{i s t} K_{0}(|t|) d t=\frac{\pi}{\left(1+s^{2}\right)^{\frac{1}{2}}}
$$

we deduce that

$$
\begin{aligned}
& \int_{-\infty}^{\infty} \cos \lambda t K_{0}(|x-t|) d t=\frac{\pi \cos \lambda x}{\left(1+\lambda^{2}\right)^{\frac{1}{2}}} \\
& \int_{-\infty}^{\infty} \sin \lambda t K_{0}(|x-t|) d t=\frac{\pi \sin \lambda x}{\left(1+\lambda^{2}\right)^{\frac{1}{2}}}
\end{aligned}
$$


It remains to obtain the bound $K_{0}(x) \leqq(\pi / 2 x)^{\frac{1}{2}} e^{-x}$ utilised in section 1 . This bound follows from the fact that $k_{u}(x)$ is, for fixed positive $x$ and $u>0$, an increasing function of $u$. Therefore $K_{0}(x) \leqq K_{\frac{1}{2}}(x)=(\pi / 2 x)^{\frac{1}{2}} e^{-x}$, after inserting the expression for $K_{\frac{1}{2}}$ stated in Magnus et al [7], p. 73.

\section{REFERENCES}

1. NOBLE, B. Methods based on the Wiener-Hopf technique, Pergamon Press, London 1958.

2. NOBLE, B. \& PETERS, A.S., A Multiplying Factor Method for the Solution of WienerHopf Equations, Proc. Edin. Math. Soc. 12 (1961) 119-122.

3. TITCHMARSH, E.C. Introduction to the Theory of Fourier Integrals, Oxford University Press, London, 1937.

4. WATSON, G.N. Theory of Bessel Functions, Second Edition, Cambridge University Press, London, 1958 .

5. LOWNDES, J.S. On Dual and Triple Integral Equations involving Modified Bessel Functions, Applicable Analysis, 6 (1977) 253-260.

6. OBERHETTINGER, F. Tables of Bessel Transforms, Springer-Verlag, New York, 1971.

7. MAGNUS, W., OBERHETTINGER, F., \& SONI, R.P. Formulas and Theorems for the Special Functions of Mathematical Physics, Springer-Verlag, New York, 1966 . 


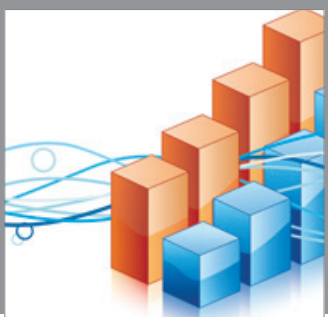

Advances in

Operations Research

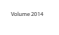

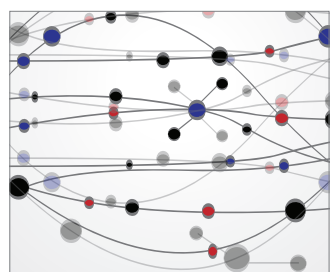

\section{The Scientific} World Journal
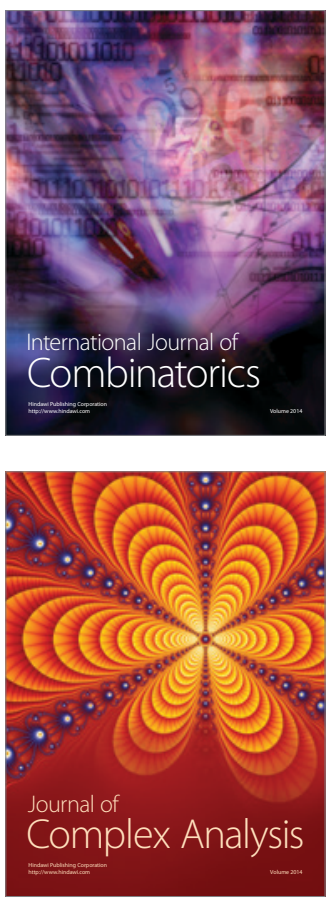

International Journal of

Mathematics and

Mathematical

Sciences
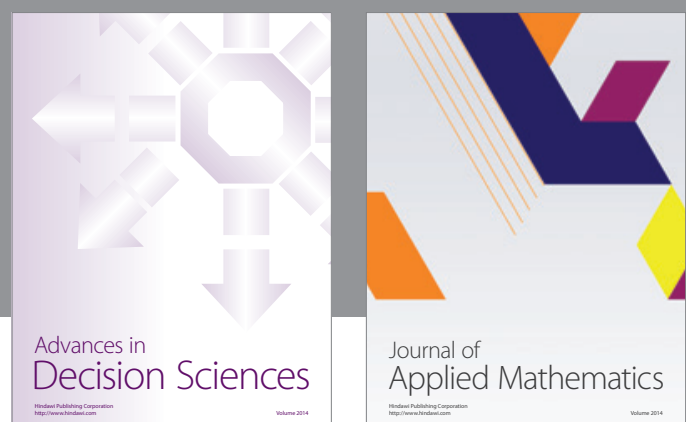

Journal of

Applied Mathematics
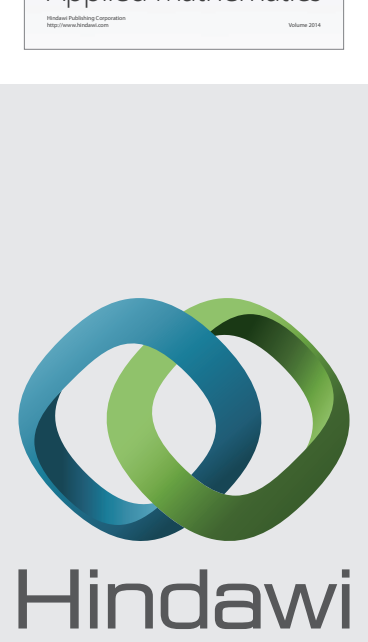

Submit your manuscripts at http://www.hindawi.com
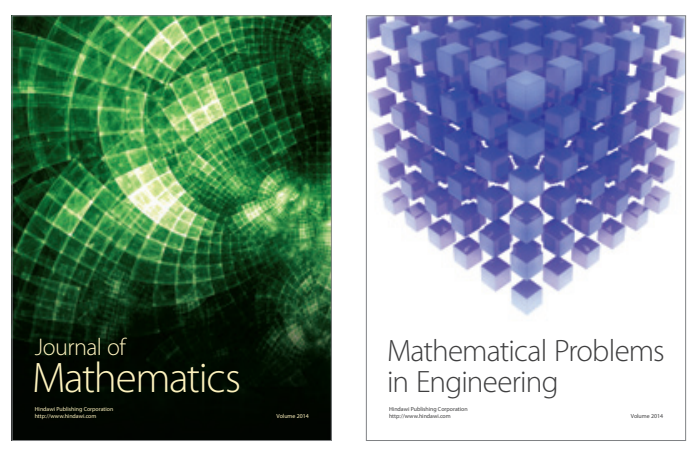

Mathematical Problems in Engineering
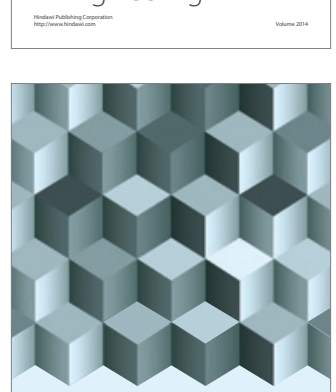

Journal of

Function Spaces
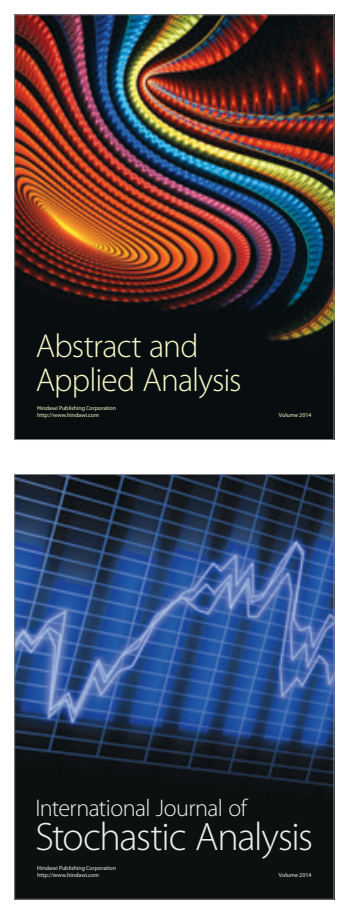

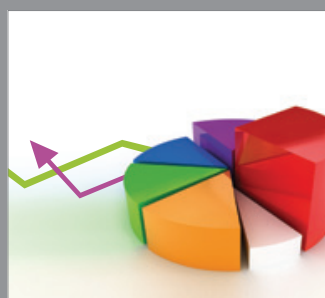

ournal of

Probability and Statistics

Promensencen
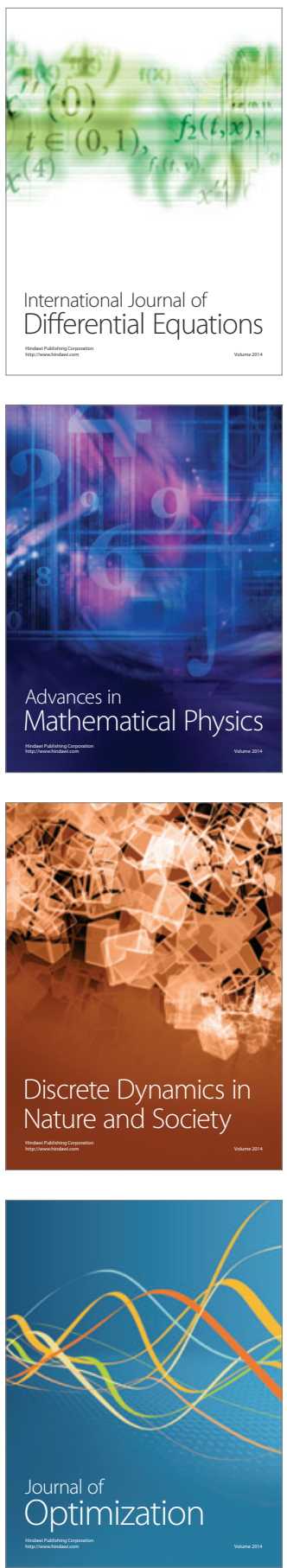